\title{
SURGERY ON PIECEWISE LINEAR MANIFOLDS AND APPLICATIONS
}

\author{
BY WILLIAM BROWDER AND MORRIS W. HIRSCH ${ }^{1}$
}

Communicated by S. Smale, March 30, 1966

1. Introduction and statement of results. In this note we indicate a method of performing surgery on piecewise linear (=PL) manifolds, and show how to prove piecewise linear analogs of theorems on the homotopy type and classification of smooth manifolds ${ }^{2}$ (Browder [1], Novikov [10], Wall [13]).

The basic principles are two: to use normal microbundles instead of normal vector bundles, and to put a differential structure $\sigma$ on a neighborhood $V$ of an embedded sphere $S \subset M$ that represents a homotopy class we wish to kill. Then smooth ambient surgery can be performed on $V_{\sigma}$, and the resulting cobordism triangulated.

Let $M_{1}, M_{2}$ be closed PL $n$-manifolds embedded in $S^{n+k}$ with normal microbundles $\nu_{1}, \nu_{2}$. A normal equivalence $b:\left(M_{1}, \nu_{1}\right) \rightarrow\left(M_{2}, \nu_{2}\right)$ is a microbundle equivalence $b: \nu_{1} \rightarrow \nu_{2}$ covering a homotopy equivalence $M_{1} \rightarrow M_{2}$.

Let $T\left(\nu_{i}\right)$ be the Thom complex of $\nu_{i}$ (see [12]), and let $c_{i} \in \pi_{n+k} T\left(\nu_{i}\right)$ be the homotopy class of the collapsing map $S^{n+k} \rightarrow T\left(\nu_{i}\right)$. We call $c_{i}$ a normal invariant for $M_{i}$. If $\partial M \neq 0$, a similar construction defines a normal invariant for $M$ as an element in $\pi_{n+k}\left(T\left(\nu_{M}\right), T\left(\nu_{M} \mid \partial M\right)\right)$.

Theorem 1. Let $X$ be a 1-connected polyhedron satisfying Poincaré duality in a dimension $n \geqq 5$. Let $\xi$ be a PL $k$-microbundle over $X$, and let $\alpha \in \pi_{n+k} T(\xi)$ be such that $h(\alpha)=\Phi(g)$, where $h: \pi_{n+k} T(\xi) \rightarrow H_{n+k} T(\xi)$ is the Hurewicz homomorphism, $\Phi: H_{n}(X) \rightarrow H_{n+k} T(\xi)$ is the Thom isomorphism, and $g \in H_{n}(X)$ is a generator. Assume $k \geqq n$. Then $X$ has the homotopy type of a closed PL n-manifold $M \subset S^{n+k}$ such that

(a) If $n$ is odd, or if $n=4 q$ and the signature of $X$ is $\left\langle L_{q}\left(\bar{p}_{1}(\xi), \ldots\right.\right.$, $\left.\left.p_{q}(\xi), g\right)\right\rangle$, then $M$ has a normal microbundle induced from $\xi$, and $\alpha$ is a normal invariant of $M$;

(b) If $n$ is even, $M$-\{point $\}$ has a normal microbundle induced from $\xi$.

${ }^{1}$ Work partially supported by the National Science Foundation (USA) and Department of Scientific and Industrial Research (UK) at the Cambridge Topology Symposium, 1964.

${ }^{2}$ We are informed that some of our results have been obtained independently by R. Lashof and M. Rothenberg. 
Theorem 1 is the PL analog of [1]; see also [10].

THEOREM 2. Let $M_{1}, M_{2}$ be PL closed 1-connected $n$-manifolds $n \geqq 5$. Then $M_{1}$ and $M_{2}$ are combinatorially equivalent if and only if there are normal microbundles $\nu_{i}(i=1,2)$ of embeddings $M_{i} \subset S^{n+k}$, with normal invariants $c_{i} \in \pi_{n+k} T\left(\nu_{\imath}\right)$, and a normal equivalence $b:\left(M_{1}, \nu_{1}\right) \rightarrow\left(M_{2}, \nu_{2}\right)$ such that $T(b)_{*}\left(c_{1}\right)=c_{2}$.

Theorem 2 is the PL analog of a theorem of Novikov [10].

Corollary. Let $M$ be a PL closed 1-connected $n$-manifold, $n \geqq 5$. Suppose the natural map $k_{\mathrm{PL}}(M) \rightarrow k_{\mathrm{Top}}(M)$ is injective and that $k_{\mathrm{PL}}(\Sigma M) \rightarrow k_{\mathrm{Top}}(\Sigma M)$ is surjective (see [8] and [9]) where $\Sigma M$ is the suspension of $M$. Then the PL structure on the underlying topological manifold $M$ is unique up to isomorphism.

Proof. Let $\nu_{1}, \nu_{2}$ be normal microbundles of two PL structures $M_{1}, M_{2}$ on $M$. By the stable uniqueness of a topological normal microbundle of $M$ [8], and the injectivity of $k_{\mathrm{PL}}(M) \rightarrow k_{\mathrm{Top}}(M)$, it follows that $\nu_{1}$ and $\nu_{2}$ are stably equivalent as PL microbundles. Let $c_{i}$ $\in \pi_{n+k} T\left(\nu_{i}\right)$ be the normal invariant of $M_{i}$. Since $M_{1}$ and $M_{2}$ are the same topological manifold, it follows that (for sufficiently large $k$ ) there is a topological microbundle equivalence $b: \nu_{1} \rightarrow \nu_{2}$ such that $T(b)_{*}\left(c_{1}\right)=c_{2}$. (The stable tubular neighborhood theorem [4], [7] is needed.) Using the surjectivity of $k_{\mathrm{PL}}(\Sigma M) \rightarrow k_{\text {Top }}(\Sigma M)$ we can choose $b$ to be a PL microbundle equivalence. The Corollary follows from Theorem 2 .

Theorem 3. Let $(X, A)$ be a polyhedral pair with both $X$ and $A$ 1-connected, satisfying Poincaré duality in a dimension $n \geqq 6$. Let $\xi$ be a PL $k$-microbundle over $X$ with $k>n$, let $e \in H_{n}(X, A)$ be a generator, and suppose there exists $\beta \in \pi_{n+k}(T(\xi), T(\xi \mid A))$ such that $h(\beta)=\Phi(e)$. Then $(X, A)$ is homotopy equivalent to $\mathrm{PL}$ manifold with boundary $(M, \partial M)$ having a normal microbundle induced from $\xi$, and having $\beta$ for a normal invariant. Moreover, $M$ is unique up to $\mathrm{PL}$ homeomorphism.

This is the PL analog of a result of Wall [13].

2. Proofs of theorems. We indicate the modification in the proofs of the analogous smooth theorems that are required in the PL case.

To prove Theorem 1 , by using the transverse regularity theorem 
of Williamson [12] we may assume that there is a PL closed $n$ manifold $N \subset S^{n+k}$ such that:

(i) if $\bar{f}: S^{n+k} \rightarrow T(\xi)$ represents $\alpha$, then $\bar{f}^{-1}(X)=N$;

(ii) if $\bar{f} \mid N=f$, then $f^{*} \xi=\nu$, the normal microbundle of $N$ in $S^{n+k}$;

(iii) $f: N \rightarrow X$ has degree 1 .

(See $[1]$.)

Main Lemma. Let $S \subset N$ be a PL embedded $p$-sphere, $p<n / 2$, such that $f \mid S: S \rightarrow X$ is null homotopic. Then there exists a PL surgery killing the homotopy class of $S$. If $N^{\prime}$ is the resulting $n$-manifold the trace of the surgery (an elementary PL cobordism $K$ between $N$ and $N^{\prime}$ ) can be embedded in $S^{n+k} \times I$ with $K \cap\left(S^{n+k} \times 0\right)=N=N \times 0$ and $K \cap\left(S^{n+k} \times 1\right)=N^{\prime}$. Moreover, $K$ has a PL normal microbundle $\eta$ in $S^{n+k} \times I$ with $\eta=g^{*} \xi$, where $g: K \rightarrow X$ extends $f: N \rightarrow X$.

Proof. Let $U \subset N$ be an open regular neighborhood of $S$. Then $f^{*} \xi|U=\nu| U$ is trivial because $f \mid U$ is null homotopic. Therefore there is a PL embedding $\phi: U \times R^{k} \rightarrow S^{n+k}$ such that $\phi(x, 0)=x$ and $\phi^{-1} N$ $=U \times 0$. By the product theorem of [5], the smoothing of $U \times R^{k}$ induced by $\phi$ is concordant to a product smoothing. In fact, there is an open neighborhood $V$ of $S$ in $N$ with $\bar{V} \subset U$, a smoothing $\sigma$ of $V$, and a piecewise differentiable isotopy $\phi_{t}: U \times R^{k} \rightarrow S^{n+k}$ such that

(i) $\phi_{0}=\phi$,

(ii) $\phi_{t}=\phi$ outside $V \times R^{k}$,

(iii) $\phi_{1} \mid V \times D^{k}$ is a smooth embedding $V_{\sigma} \times D^{k} \rightarrow S^{n+k}$.

Observe now that $\phi_{1}\left(V_{\sigma} \times 0\right)$ is a smooth submanifold of $S^{n+k}$ and $\phi_{1}$ provides a trivialization of its normal vector bundle. Let $V^{\prime} \subset V_{\sigma}$ be a smooth closed neighborhood of $S$, and put $W_{0}=\phi_{1}\left(V^{\prime} \times 0\right)$. Let $W_{1} \subset S^{n+k}$ be the smooth submanifold obtained from $W_{0}$ by a smooth surgery killing the homotopy class of $\phi(S \times 0)$. By Haefliger [2] the trace of the surgery is a cobordism $L$ between $W_{0}$ and $W_{1}$ smoothly embedded in $S^{n+k} \times I$ such that $\partial L=W_{0} \times 0 \cup\left(\partial W_{0}\right) \times I \cup W_{1} \times 1$, and such that the embedding is the product embedding in a neighborhood of $\partial W_{0} \times I$. Furthermore, the map $f^{\prime}: W_{0} \times 0 \cup\left(\partial W_{0}\right) \times I \rightarrow X$, defined to be the composition

$$
\left(W_{0} \times 0\right) \cup\left(\partial W_{0}\right) \times I \rightarrow W_{0} \stackrel{\phi_{1}^{-1}}{\longrightarrow} N \rightarrow X
$$

extends to $f^{\prime \prime}: L \rightarrow X$ such that $f^{\prime \prime * \xi}$ is the normal bundle of $L$ in $S^{n+k} \times I$. 
The cobordism $L$ and the product cobordism $\left(N-\right.$ int $\left.V^{\prime}\right) \times I$ fit totogether to form a cobordism $K_{1} \subset S^{n+k} \times I$ between $N \times 0$ and $\left(\left(N-\right.\right.$ int $\left.\left.V^{\prime}\right) \cup W_{1}\right) \times 1$. The composition

$$
\left(N-\operatorname{int} V^{\prime}\right) \times I \rightarrow N \stackrel{f}{\rightarrow} X
$$

and $f^{\prime \prime}: L \rightarrow X$ fit together to give a map $g: K_{1} \rightarrow X$. The microbundle $\nu$ extends to a microbundle $\eta$ over $K_{1}$ that coincides with $\nu$ over $N \times 0$, with $\nu \times I$ over $\left(N-\right.$ int $\left.V^{\prime}\right) \times I$, and such that $\phi_{1}$ is a trivialization of $\eta \mid W_{1} \times 1$. In fact, $\eta=g^{*} \xi$. The isotopy $\phi_{t}$ provides an embedding $G: E \eta \rightarrow S^{n+k} \times I$ of the total space $\eta$ which is the identity on $E \nu$. Consider $G$ as a smooth triangulation of an open subset of $S^{n+k} \times I$. Whitehead's triangulation theorems show that there is a neighborhood $E_{0}$ of the zero section of $\eta$ and a homeomorphism $H$ of $S^{n+k} \times I$ such that $H G \mid E_{0}$ is PL, and $H \mid S^{n+k} \times 0$ is the identity. Thus $K$ $=H G\left(K_{1}\right)$ is the desired cobordism. This completes the proof of the Main Lemma.

The proof of Theorem 1 proceeds as in the smooth case if $n$ is odd. If $n$ is even, we proceed until we have an $N$ such that $f: N \rightarrow X$ is an isomorphism in homotopy below the middle dimension. Following the procedure of the proof of the main lemma, we find just as in the smooth case that the obstruction $c$ to surgery is a signature or Kervaire-Arf invariant of the intersection quadratic form on the kernel $K_{r}$ of $f_{*}$ in $H_{r}(N), 2 r=n$. If the signature of $X$ is as in (a) of Theorem 1 , then $c=0$; otherwise $c \equiv 0 \bmod 8$. (To see this, recall that a nonsingular quadratic form taking only even values has signature divisible by 8 . It suffices to prove $x \# x=0$ for $x \in \operatorname{ker}\left(f_{*} \mid H_{r}\left(N ; Z_{2}\right)\right)$. If $P: H^{*}\left(N ; Z_{2}\right) \rightarrow H_{*}\left(N ; Z_{2}\right)$ is Poincaré duality, then $x \# y=\left\langle P^{-1} x \cup P^{-1} y\right.$, $N\rangle$ for $x, y \in H_{r}\left(N ; Z_{2}\right)$. Let $P^{-1} x=z$. Then $x \# x=\langle\mathrm{Sq} z, N\rangle=\left\langle z \cup U_{N}\right.$, $N\rangle$ where $U_{N} \in H^{*}(N)$ is the total Wu class. Since $\mathrm{Sq}^{-1} U_{N}=W_{N}$ (the total Stiefel-Whitney class of $N$ ), if we define $U_{X}=\mathrm{Sq}^{-1} W(\xi)^{-1}$ it follows that $U_{N}=f^{*} U_{X}$, and $x \# x=\left\langle Z \cup f^{*} U_{X}, N\right\rangle=x \# P f^{*} U_{X}$. By [1], $K_{r}$ is orthogonal to $P f^{*}\left(H^{*}(X)\right)$. Hence $x \# x=0$.)

There exists an oriented PL closed $(r-1)$-connected $2 r$-manifold $P$ with signature -8 if $r=2 q$, and with Kervaire-Arf invariant 1 if $r=2 q+1$. Moreover $P$ - $\{$ point $\}$ is parallelizably smoothable. It follows [3] that there is a PL embedding $P \subset S^{2 r+2}$ having a trivial normal bundle on $P_{0}$ (the complement of a highest dimensional cell). Therefore the connected sum $N \# P$ embeds in $S^{n+k}$ with a normal microbundle $\nu^{\prime}$ on $(N \# P)_{0}$ which coincides with the normal microbundle $\nu$ of $N$ on $N_{0}$, and which is trivial on the rest of $(N \# P)_{0}$. 
Let $N^{\prime}=N \# P$ if $r=2 q+1$, and let $N^{\prime}$ be the connected sum of $N$ with $c / 8$ copies of $P$ if $r=2 q$. Define $f^{\prime}: N^{\prime} \rightarrow X$ by $f^{\prime} \mid N_{0}=f$, and $f \mid N^{\prime}-N_{0}$ constant. Since $\nu^{\prime} \mid N^{\prime}-N_{0}$ is trivial, $f^{\prime}$ is covered by a microbundle map $\nu^{\prime} \rightarrow \xi$. The obstruction to surgery on $N^{\prime}$ now vanishes. Hence by surgery we obtain a manifold $M \subset S^{n+k}$ with a normal microbundle $\nu$ on $M_{0}$ and a homotopy equivalence $f: M \rightarrow X$ such that $f \mid M_{0}$ is covered by a microbundle map $\nu \rightarrow \xi$.

Alternatively, in the middle dimension we could use the method of $[14]$.

Theorem 2 is proved in a similar way, using the same trick to extend Novikov's proof to the PL case. Since for $n \geqq 5$ any PL homotopy sphere $T$ is a combinatorial sphere (Smale [11]), the conclusion of the smooth case, that $M_{1} \# T=M_{2}$ becomes $M_{1}=M_{2}$ in the PL case.

For Theorem 3 we imitate the proof of Theorem 2 of Wall [13] with the following modification of the immersion argument of [13]. Given a PL map $f: D^{k+1} \rightarrow M^{2 k+1}$ (in the notation of [13]), assume that $f$ has generic singularities. It follows that $H^{i} f\left(D^{k+1}\right)=0$ for $i>2$. Since $\Gamma_{i}=0$ for $i \leqq 2$, it follows from [5] that a neighborhood $V$ of $f\left(D^{k+1}\right)$ in $M^{2 k+1}$ has a smoothing $\sigma$. Then we approximate $f$ by a smooth map into $V_{\sigma}$ and proceed as in [13].

\section{BIBLIOGRAPHY}

1. W. Browder, Homotopy type of differentiable manifolds, Proc. Aarhus Symposium, 1962.

2. A. Haefliger, Knotted (4k-1)-spheres in 6k-space, Ann. of Math. (2) 75 (1962), 452-466.

3. M. W. Hirsch, On imbedding differentiable manifolds in euclidean space, Ann.of Math. (2) 73 (1961), 566-571.

4. - On normal microbundles, University of California, 1966 (mimeographed) Topology (to appear).

5. - Obstructions to smoothing manifolds and maps, Bull. Amer. Math. Soc. 69 (1963), 352-356.

6. M. Kervaire and J. Milnor, Groups of homotopy spheres. I, Ann. of Math. (2) 77 (1963), 504-537.

7. R. Lashof and M. Rothenberg, Microbundles and smoothing, Topology 3 (1965), 357-388.

8. J. Milnor, Microbundles. I, Topology 3 (1964), Suppl. 1, 53-80.

9. - Microbundles and differentiable structures, Princeton University, Princeton, N. J., 1961. (mimeographed)

10. S. P. Novikov, Diffeomorphisms of simply connected manifolds, Soviet Math. Dokl. 3 (1962), 540-543.

11. S. Smale, On the structure of manifolds, Amer. J. Math. 84 (1962), 387-399.

12. R. Williamson, Cobordism of combinatorial manifolds, Ann. of Math. (2) 83 (1966), 1-83. 
13. C. T. C. Wall, An extension of results of Novikov and Browder, Amer. J. Math. 88 (1966), 20-32.

14. Y.-F. Wong, $A$ theorem on homotopically equivalent $(2 k+1)$-manifolds, Proc. Amer. Math. Soc. 16 (1965), 1022-1025.

\section{Princeton University and}

University of California, Berkeley 